\title{
Understanding adhesion at as-deposited interfaces from ab initio thermodynamics of deposition growth: thin-film alumina on titanium carbide
}

\author{
Jochen Rohren* and Per Hyldgaard \\ BioNano Systems Laboratory, Department of Microtechnology, \\ MC2, Chalmers University of Technology, SE-412 96 Gothenburg
}

(Dated: September 13, 2021)

\begin{abstract}
We investigate the chemical composition and adhesion of chemical vapour deposited thin-film alumina on $\mathrm{TiC}$ using and extending a recently proposed nonequilibrium method of ab initio thermodynamics of deposition growth (AIT-DG) [Rohrer J and Hyldgaard P 2010 Phys. Rev. B 82 045415]. A previous study of this system [Rohrer J, Ruberto C and Hyldgaard P 2010 J. Phys.: Condens. Matter 22 015004] found that use of equilibrium thermodynamics leads to predictions of a non-binding $\mathrm{TiC} /$ alumina interface, despite the industrial use as a wear-resistant coating. This discrepancy between equilibrium theory and experiment is resolved by the AIT-DG method which predicts interfaces with strong adhesion. The AIT-DG method combines density functional theory calculations, rate-equation modelling of the pressure evolution of the deposition environment and thermochemical data. The AIT-DG method was previously used to predict prevalent terminations of growing or as-deposited surfaces of binary materials. Here we extent the method to predict surface and interface compositions of growing or as-deposited thin films on a substrate and find that inclusion of the nonequilibrium deposition environment has important implications for the nature of buried interfaces.
\end{abstract}

\section{INTRODUCTION}

Interfaces and surfaces are present in practically all devices and their detailed structure is typically crucial for the overall device functionality [1]6]. Understanding and ultimately controlling the thin-film deposition, the chemical composition and the adhesion at interfaces [7-18 is a very desirable goal of industrial and scientific research. Characterisation of atomic structure and binding at interfaces is a fundamental step towards this goal.

Atomistic modelling of materials 19 27 using methods based on ab-initio density functional theory (DFT) allows for a detailed understanding of structure at the atomic and electronic level. Developing reliable modelling methods is of particular value for characterisation of interfaces which are buried inside materials and therefore difficult to characterise experimentally with atomic resolution [20, 28 31]. Atomistic modelling has a potential to accelerate innovation, for example, in the development of coatings and in the design of functional surface and interface materials [32. A key element of a reliable method is a proper treatment of thermodynamic effects of a surrounding environment during creation of thin films and interfaces.

Until recently, ab-initio thermodynamics (AIT) methods were essentially methods of surface equilibrium (and here denoted as AIT-SE). These methods have focused on oxide surfaces 33, 34 or metal/oxide interfaces 35 assuming equilibrium between the oxide surface (interface) and $\mathrm{O}_{2}$ in an $\mathrm{O}_{2}$-dominated, e.g., ambient environment. Oxide surfaces are in direct contact with the environment and the oxygen content of this environment will therefore

\footnotetext{
*Electronic address: rohrer@chalmers.se
}

have a strong influence on the termination of the oxide. However, it is by no means clear how a surrounding could easily influence the composition at interfaces (which are by definition buried and insulated from the gaseous environment). In fact, we have shown [36] that adapting and applying this AIT-SE method to the TiC/alumina interface predicts a structure and composition that possesses essentially no binding across the interface. This result is evidently in conflict with the actual use of chemical vapour deposited (CVD) $\mathrm{TiC}$ /alumina multilayers as wear-resistant coating on cemented-carbide cutting-tools [37, 38.

The present paper demonstrates that realistic descriptions of deposition environments are crucial for characterising thin-film and interface compositions and, as a consequence, adhesion to the underlying substrate. We show how effects of a steady-state deposition environment (for example CVD) can be embedded into atomistic modelling. We employ a method of ab-initio thermodynamics of deposition growth (AIT-DG) 39, but extend it here to suit the more complex problem of understanding thin-film formation and corresponding as-deposited interfaces. We focus our discussion on $\mathrm{TiC} /$ alumina.

The fundamental strategy is to compare free energies of reaction associated with thin-film configurations that differ in their detailed chemical composition. The key elements of the method are: (i) analysis of free energies of reaction $G_{\mathrm{r}}$ and (ii) use of rate-equation modelling for the pressure evolution of the deposition environment. The key variables that determine $G_{\mathrm{r}}$ are the partial pressures of the various constituents of the environment. Assuming a steady state, all partial pressures can be expressed in terms of a few rates. No assumptions about equilibrium between some of the species enter into this analysis. We point out that a steady state does not necessarily correspond to a state, sometimes described as dynamic equi- 


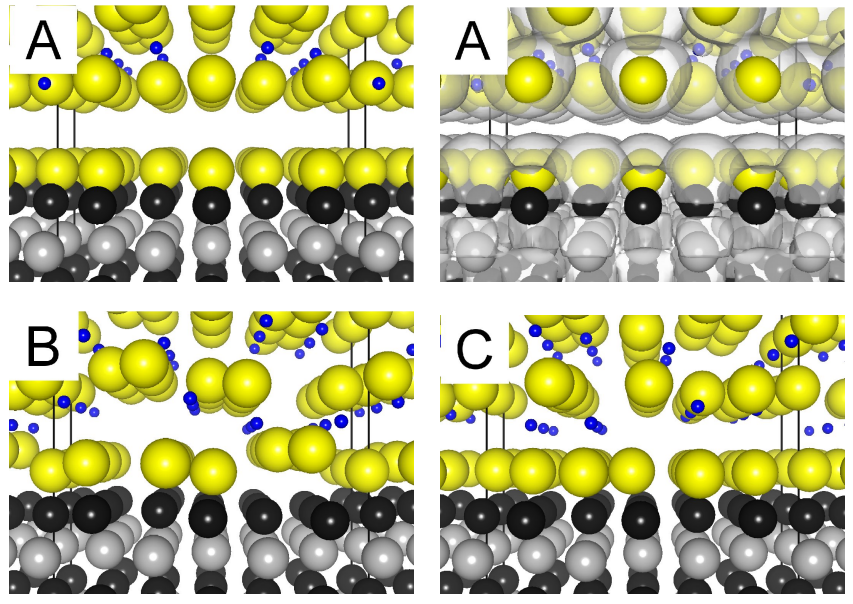

FIG. 1: (Colour online) Structure of interfaces between TiC and various thin-film alumina overlayers. Colour coding: Ti = black, $\mathrm{C}=$ gray, $\mathrm{Al}=$ blue (black, small balls) and $\mathrm{O}=$ yellow (light gray). The overlayers can be sorted into classes of thin-films with different nature of interfaces (A, B, C) and possessing different deviations from the full $\mathrm{Al}_{2} \mathrm{O}_{3}$ stoichiometry (here, $\mathrm{Al}$ deficiency only). The interface class predicted by the equilibrium AIT-SE method is shown in the top panels. Its non-binding character is obvious from the geometric structure (left) and electronic density (right), and in conflict with the wear-resistance of $\mathrm{TiC} /$ alumina coatings. The set of bottom panels shows alternative classes of thin-film alumina on TiC. These show stronger adhesion at the interface and have correct nature according to industrial use. They are found unstable according AIT-SE but stable according to the present nonequilibrium account, AIT-DG.

librium [40, where the system is assumed to gain no free energy by the deposition of stoichiometric alumina. The method also describes the evolving system in a certain range where dynamic equilibrium is not maintained.

The paper is organised as follows. In Section [I] we summarise the results of a previous structure search and equilibrium-thermodynamic analysis for $\mathrm{TiC} /$ alumina interfaces [36]. This section highlights the inconsistency in adhesion properties of the predictions made with the AIT-SE method. In Section III we give a general motivation for the use of Gibbs free energies of reaction to predict the prevalence of thin-film classes and chemical compositions at as-deposited interfaces. Section IV presents a simple model that describes the environment used for chemical vapour deposition of alumina on TiC. Section $\nabla$ contains the details for the evaluation of Gibbs free energies of reaction for $\mathrm{TiC} /$ alumina interfaces. In Section VI, we present and discuss our results. We summarise our work and conclude in Section VII]

\section{BACKGROUND}

In Ref. 36, we have presented an $a b$ initio structure search for thin-film alumina on TiC. We have considered thin-film alumina configurations with different thicknesses and various chemical compositions. The configurations can be characterised by two numbers $t$ and $\Delta_{\mathrm{Al}}$. Here, $t$ is the film thickness in terms of the number of $\mathrm{O}$ layers and $\Delta_{\mathrm{Al}}=N_{\mathrm{Al}}-2 / 3 N_{\mathrm{O}}$ is the number of excess $\left(\Delta_{\mathrm{Al}}>0\right)$ or deficiency $\left(\Delta_{\mathrm{Al}}<0\right)$ in the number of $\mathrm{Al}$ atoms.

All configurations approach the full stoichiometry in the thick-film limit. They essentially differ in chemical composition only at the interface, surface, or both. We therefore, in general, group the configurations into interface classes according to their corresponding value of $\Delta_{\mathrm{Al}}$. Interface class $\mathrm{A}$ corresponds to $\Delta_{\mathrm{Al}}=-4$, interface class $\mathrm{B}$ corresponds to $\Delta_{\mathrm{Al}}=-2$ and interface class $\mathrm{C}$ corresponds to $\Delta_{\mathrm{Al}}=0$. Other values of $\Delta_{\mathrm{Al}}$ were not considered.

Figure 1 presents energetically optimised TiC/alumina configurations for all three interface classes. The set of top panels details of the atomic (left) and electronic structure (right) at the interface class A. Straightforward adaption and use of the equilibrium AIT-SE method 33 35 identifies interface class $\mathrm{A}$ as thermodynamically stable over a wide range of temperatures and $\mathrm{O}_{2}$ pressures, see Ref. 36. However, no appreciable adhesion is found at interfaces of this type. These interfaces separate into a $\mathrm{TiC}$ substrate covered with a full layer of $\mathrm{O}(\mathrm{TiC} / \mathrm{O})$ and a fully stoichiometric alumina overlayer. The electronic structure shows that the electron density essentially vanishes between the $\mathrm{TiC} / \mathrm{O}$ and the alumina, ruling out a significant covalent binding. Furthermore, the $\mathrm{Al}$ ions at the interface relax into the first $\mathrm{O}$ layer above the $\mathrm{TiC} / \mathrm{O}$, ruling out significant ionic binding.

In the present work we quantify the (lack of) adhesion by calculating the ideal work of adhesion as $W_{\text {adh }}=$ $\left(E_{\text {substrate }}+E_{\text {alumina }}-E_{\mathrm{TiC} / \text { alumina }}\right) / S$. Here $E_{\text {substrate }}$ and $E_{\text {alumina }}$ are the energies of the isolated relaxed substrate ( $\mathrm{TiC}$ for interface class $\mathrm{B}$ and $\mathrm{C}$ or $\mathrm{TiC} / \mathrm{O}$ for interface class A) and the isolated relaxed alumina film (with in-plane lattice parameters constraint to the surface lattice of $\mathrm{TiC}$ ). $E_{\mathrm{TiC} / \text { alumina }}$ is the energy of the relaxed interface and $S$ is the area of the contact surface. For interface class A we find a nonquantifiable (vanishing) value of $W_{\text {adh }}$, comparable to the uncertainty in the force relaxation in the underlying GGA calculations (described in Ref. 36).

The non-binding character at interface class A is clearly in conflict with the wear-resistance of $\mathrm{TiC} /$ alumina multilayers [37. We attribute this conflict to the fact that alumina does neither maintain equilibrium with $\mathrm{O}_{2}$ during deposition ${ }^{1}$ nor can one expect that it reaches such equilibrium after being removed from the deposition chamber. This true for the present focus on thin films and even more so for thicker overlayers.

1 At best the alumina may maintain dynamic equilibrium with a number of gases in the surrounding. However, as we will also discuss below, even this assumption is too optimistic for the case of CVD of alumina on TiC. 


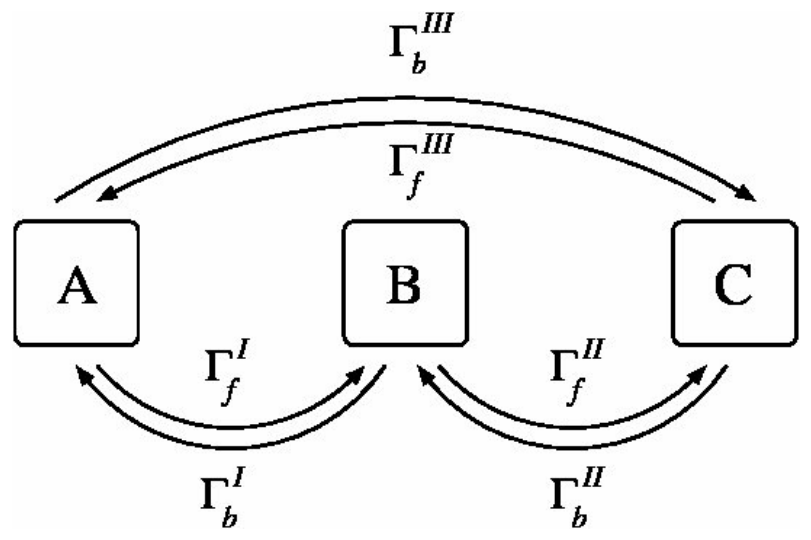

FIG. 2: Schematics of elementary growth processes in an ensemble of systems that can be organised into three different classes A, B and C. The members of these classes could be alumina films (of various thicknesses but) sorted by their balance of $\mathrm{Al}$ and $\mathrm{O}$ atoms and therefore having different surfaces and/or interfaces to the substrate. The resulting interface classes may exhibit a very different nature of adhesion. Combining chemical reaction theory with a rate-equation description of the system allows us to predict the probabilities for realisation of class A, B and C during steady-state growth.

According to the AIT-SE method, interface class B (see bottom left of Figure 1) is predicted to be stable only under extreme conditions, whereas interface class C (see bottom right panel) is predicted unstable over the whole range of allowed values of $\mathrm{O}$ chemical potentials [36. However, the works of adhesion associated with these interface classes, $W_{\mathrm{adh}}=7.4 \mathrm{~J} / \mathrm{m}^{2}$ for interface class $\mathrm{B}$ and $W_{\text {adh }}=7.3 \mathrm{~J} / \mathrm{m}^{2}$ for interface class $\mathrm{C}$, are in much better agreement with the wear-resistance of the material. We show below that the conditions prevailing during CVD of alumina allow for nucleation of either one of these binding interface classes B and C.

\section{AB INITIO THERMODYNAMICS OF DEPOSITION GROWTH}

In Ref. 39 we have introduced the AIT-DG method and demonstrated that the Gibbs free energy of reaction can be used as a predictor for the prevalence of the chemical composition at a growing surface. This was done for a binary material with two possible surface terminations. Here we extend this nonequilibrium description to the problem of identifying the composition of growing overlayers which exhibit both a surface and an interface to the substrate on which they are deposited. We follow a similar line of argumentation as in Ref. 39 and combine chemical reaction theory [40, with a rate-equation description of the probabilities for finding any of the possible film compositions.

Figure 2 illustrates a collection of systems (e.g. an ensemble of growing thin films) that are grouped into three thin-film and interface classes A, B and C. The members of the classes can have different film thicknesses but are sorted according to their chemical composition in terms of excess or deficiency atoms of a specific species. Members of different classes are allowed to transform into members of other classes via the three reactions labeled as $I, I I$, and $I I I$, all being characterised by forward and backward rates $\Gamma_{f}^{i}$ and $\Gamma_{b}^{i}(i=I, I I, I I I)$. Growth of, for example, a film of class A results by a net flow along the reaction chain, $\mathrm{A} \rightarrow \mathrm{B} \rightarrow \mathrm{C} \rightarrow \mathrm{A}$.

Chemical reaction theory relates the forward and backward rates to the inverse temperature $\beta$ (in units of energy) and the Gibbs free energy of reaction by $\beta \Delta G_{r}^{i}=$ $-\ln \Gamma_{f}^{i} / \Gamma_{b}^{i}$. At the same time, the probability for a random member of the ensemble to belong to one of the three classes is described in terms of rate equations,

$$
\begin{aligned}
& \partial_{t} P_{\mathrm{A}}=-\left(\Gamma_{f}^{I}+\Gamma_{b}^{I I I}\right) P_{\mathrm{A}}+\Gamma_{b}^{I} P_{\mathrm{B}}+\Gamma_{f}^{I I I} P_{\mathrm{C}} \\
& \partial_{t} P_{\mathrm{B}}=\Gamma_{f}^{I} P_{\mathrm{A}}-\left(\Gamma_{b}^{I}+\Gamma_{f}^{I I}\right) P_{\mathrm{B}}+\Gamma_{b}^{I I} P_{\mathrm{C}} \\
& \partial_{t} P_{\mathrm{C}}=\Gamma_{b}^{I I I} P_{\mathrm{A}}+\Gamma_{f}^{I I} P_{\mathrm{B}}-\left(\Gamma_{f}^{I I I}+\Gamma_{b}^{I I}\right) P_{\mathrm{C}} .
\end{aligned}
$$

This follows from a straightforward generalisation of the analysis presented in Ref. 39. The steady-state solutions for the probabilities can be expressed as ratios of sums of products of reaction rates, for example,

$$
\frac{P_{\mathrm{A}}}{P_{\mathrm{B}}}=\frac{\Gamma_{b}^{I} \Gamma_{b}^{I I}+\Gamma_{b}^{I} \Gamma_{f}^{I I I}+\Gamma_{f}^{I I} \Gamma_{f}^{I I I}}{\Gamma_{b}^{I I} \Gamma_{b}^{I I I}+\Gamma_{b}^{I I} \Gamma_{f}^{I}+\Gamma_{f}^{I} \Gamma_{f}^{I I}} .
$$

We use the differences in Gibbs free energies of reaction as an approximate predictor for the prevalence of the different classes of films and interfaces,

$$
\begin{gathered}
\beta \Delta G_{r}^{I}=\ln \frac{\Gamma_{b}^{I}}{\Gamma_{f}^{I}} \approx \ln \frac{P_{\mathrm{A}}}{P_{\mathrm{B}}} \\
\beta \Delta G_{r}^{I I}=\ln \frac{\Gamma_{b}^{I I}}{\Gamma_{f}^{I I}} \approx \ln \frac{P_{\mathrm{B}}}{P_{\mathrm{C}}} \\
\beta \Delta G_{r}^{I I I}=\ln \frac{\Gamma_{b}^{I I}}{\Gamma_{f}^{I I I}} \approx \ln \frac{P_{\mathrm{C}}}{P_{\mathrm{A}}} .
\end{gathered}
$$

The evaluations of relative probabilities (3) are exact in the limit where $\Delta G_{r}^{I}+\Delta G_{r}^{I I}+\Delta G_{r}^{I I I}=0$ or, equivalently, $\Gamma_{f}^{I} \Gamma_{f}^{I I} \Gamma_{f}^{I I I}=\Gamma_{b}^{I} \Gamma_{b}^{I I} \Gamma_{b}^{I I I}$. This limit corresponds to dynamic equilibrium in stoichiometric growth of thin films (irrespective to which interface class they belong). We note that these predictors [and the full evaluation (2p] are beyond a simple assumption of detailed balance because the probabilities are steady-state but not equilibrium probabilities.

With any use of the presented AIT-DG modelling, one should always check the quality of the approximative predictors (3). The appendix provides the theoretical framework for such a test in the case of alumina thin-film deposition and interface formation on $\mathrm{TiC}$. 


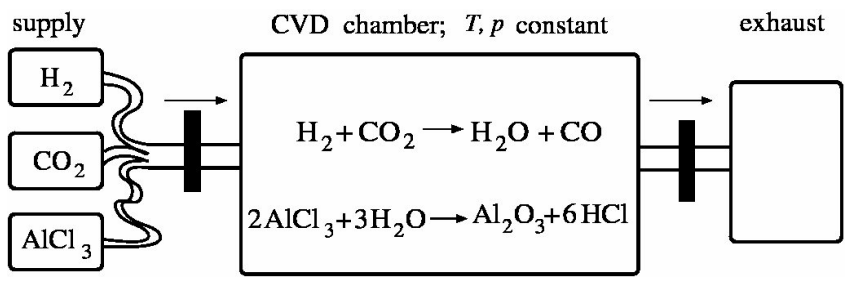

FIG. 3: Schematic model of chemical vapour deposition of alumina. $\mathrm{A} \mathrm{H}_{2}-\mathrm{AlCl}_{3}-\mathrm{CO}_{2}$ gas mixture with relative concentrations $c_{\mathrm{H}_{2}}, c_{\mathrm{AlCl}_{3}}$ and $c_{\mathrm{CO}_{2}}$ is supplied at rate $R_{\mathrm{S}}$ to a hot reaction chamber which is kept at constant temperature. At the same time, there is a continuous exhaust of gases at rate $R_{\mathrm{E}}$, also keeping the pressure constant. Including the reactions (4a) and (4b), taking place inside the chamber, we model this system by the set of rate equations (5). The resulting steady-state partial pressures can be used to determine the free energies of reactions (8) for different $\mathrm{TiC} /$ alumina systems.

\section{MODELLING OF CVD OF ALUMINA}

\section{A. Rate-equation modelling}

Figure 3 illustrates the CVD process utilised for alumina growth on $\mathrm{TiC}$. $\mathrm{A} \mathrm{H}_{2}-\mathrm{AlCl}_{3}-\mathrm{CO}_{2}$ supply gas mixture with relative concentrations $c_{i}$ is supplied to a hot chamber at rate $R_{\mathrm{S}}$. Inside the chamber water and alumina form (in parallel) at rates $R_{\mathrm{H}_{2} \mathrm{O}}$ and $R_{\mathrm{Al}_{2} \mathrm{O}_{3}}$ according to 41 .

$$
\begin{gathered}
\mathrm{H}_{2}+\mathrm{CO}_{2} \stackrel{R_{\mathrm{H}_{2} \mathrm{O}}}{\longrightarrow} \mathrm{H}_{2} \mathrm{O}+\mathrm{CO}, \\
2 \mathrm{AlCl}_{3}+3 \mathrm{H}_{2} \mathrm{O} \stackrel{R_{\mathrm{Al}_{2} \mathrm{O}_{3}}}{\longrightarrow} \mathrm{Al}_{2} \mathrm{O}_{3}+6 \mathrm{HCl} .
\end{gathered}
$$

The total pressure inside the chamber is kept at a constant value by a continuous exhaust at rate $R_{\mathrm{E}}$ of both reaction products and unused supply gases.

We describe the evolution of the environment by individual partial (ideal gas) pressures with a coupled set of rate equations,

$$
\partial_{t} p_{i} \propto c_{i} R_{\mathrm{S}}-\frac{p_{i}}{p} R_{\mathrm{E}}+\nu_{i}^{\mathrm{H}_{2} \mathrm{O}} R_{\mathrm{H}_{2} \mathrm{O}}+\nu_{i}^{\mathrm{Al}_{2} \mathrm{O}_{3}} R_{\mathrm{Al}_{2} \mathrm{O}_{3}} .
$$

Here, $p_{i}=p_{i}(t)$ is the momentary pressure of chemical species $i$ inside the reaction chamber, $p=p(t)=\sum_{i} p_{i}(t)$ is the momentary total pressure, $c_{i}$ is the concentration of the chemical species $i$ in the supply gas, and $\nu_{i}^{\mathrm{H}_{2} \mathrm{O}}$ and $\nu_{i}^{\mathrm{Al}_{2} \mathrm{O}_{3}}$ are the stoichiometric coefficients ${ }^{2}$ of the chemical species $i$ in reaction $4 \mathrm{a}$ and $4 \mathrm{~b}$, respectively.

The resulting steady-state partial pressures $\left(\partial_{t} p_{i}(t)=\right.$ $0, p(t)=p=$ const.) can be expressed in terms of

2 Stoichiometric coefficients are counted negative if a species is consumed and positive if a species is produced in a reaction. scaled reaction rates $r_{\mathrm{H}_{2} \mathrm{O}}=R_{\mathrm{H}_{2} \mathrm{O}} / R_{\mathrm{S}}$ and $r_{\mathrm{Al}_{2} \mathrm{O}_{3}}=$ $R_{\mathrm{Al}_{2} \mathrm{O}_{3}} / R_{\mathrm{S}}$,

$$
p_{i}=p \frac{c_{i}+r_{\mathrm{H}_{2} \mathrm{O}} \nu_{i}^{\mathrm{H}_{2} \mathrm{O}}+r_{\mathrm{Al}_{2} \mathrm{O}_{3}} \nu_{i}^{\mathrm{Al}_{2} \mathrm{O}_{3}}}{1+r_{\mathrm{Al}_{2} \mathrm{O}_{3}}} .
$$

This result allows us to determine free energies of reaction (see below) from a few experimentally controllable $\left(p, T, R_{\mathrm{S}}\right)$ or at least measurable $\left(R_{\mathrm{H}_{2} \mathrm{O}}, R_{\mathrm{Al}_{2} \mathrm{O}_{3}}, R_{\mathrm{E}}\right)$ quantities.

\section{B. Limits on reaction rates}

The reaction rates possess natural bounds that cannot be exceeded in steady state. First, for each reaction an upper bound for the reaction rate is given by the condition of dynamic equilibrium in this reaction. Reaction (4a) can proceed from left to right only if the chemical potentials fulfil $\mu_{\mathrm{H}_{2}}+\mu_{\mathrm{CO}_{2}} \geq \mu_{\mathrm{H}_{2} \mathrm{O}}+\mu_{\mathrm{CO}}$. Similarly, reaction $4 \mathrm{~b}$ requires that $2 \mu_{\mathrm{AlCl}_{3}}+3 \mu_{\mathrm{H}_{2} \mathrm{O}} \geq \Delta G_{\mathrm{Al}_{2} \mathrm{O}_{3}}+$ $6 \mu_{\mathrm{HCl}}$, where $\Delta G_{\mathrm{Al}_{2} \mathrm{O}_{3}}$ is the gain in free energy per stoichiometric formula of $\mathrm{Al}_{2} \mathrm{O}_{3}$. Dynamic equilibrium in these reactions is reached if equality holds which corresponds to a specific values of $R_{\mathrm{H}_{2} \mathrm{O}}$ and $R_{\mathrm{Al}_{2} \mathrm{O}_{3}}$.

In the present case another bound is found by comparing reactions $4 \mathrm{a}$ and $(4 \mathrm{~b})$. The latter reaction requires three units of $\mathrm{H}_{2} \mathrm{O}$, while the former produces only one. Thus, in steady state, $R_{\mathrm{Al}_{2} \mathrm{O}_{3}} \leq 3 R_{\mathrm{H}_{2} \mathrm{O}}=R_{\mathrm{Al}_{2} \mathrm{O}_{3}}^{\max }$. However, we do not expect our model to be applicable in the the limit where $R_{\mathrm{Al}_{2} \mathrm{O}_{3}} \rightarrow R_{\mathrm{Al}_{2} \mathrm{O}_{3}}^{\max }$. In this limit alumina deposition becomes instantaneous and on average, the water pressure will vanish. The ideal-gas description of the environment will therefore be inappropriate and kinetic aspects become dominating. We choose to consider situations where $R_{\mathrm{Al}_{2} \mathrm{O}_{3}}$ is sufficiently separated from $R_{\mathrm{Al}_{2} \mathrm{O}_{3}}^{\max }$.

\section{AB INITIO EVALUATION OF GIBBS FREE ENERGIES}

The Gibbs free energies of reaction are calculated as follows. We consider a general $\mathrm{Al}_{M} \mathrm{O}_{N}$ film as the product of a (hypothetical) chemical reaction starting from the substrate (where $M=N=0$ ). Reaction (4b) describes stoichiometric solidification of alumina. In addition, the CVD environment enables the following reaction pathways for non-stoichiometric deposition, ${ }^{3}$

$$
\begin{aligned}
\mathrm{AlCl}_{3}+\frac{3}{2} \mathrm{H}_{2} & \rightarrow \mathrm{Al}_{\text {film }}+3 \mathrm{HCl} \\
\mathrm{H}_{2} \mathrm{O} & \rightarrow \mathrm{O}_{\text {film }}+\mathrm{H}_{2} .
\end{aligned}
$$

3 We do not include the reaction $\mathrm{CO}_{2} \rightarrow \mathrm{O}_{\text {exc }}+\mathrm{CO}$ since the associated free energy of reaction is higher than that associated with $7 \mathrm{~b}$. 
The label 'film' indicates the incorporation into the alumina film on the substrate.

We define the free energy of reaction, $G_{\mathrm{r}}^{M, N}$, associated with the (hypothetical direct) deposition of a general $\mathrm{Al}_{M} \mathrm{O}_{N}$ film,

$$
\begin{aligned}
G_{\mathrm{r}}^{M, N} & =G_{\mathrm{TiC} / \mathrm{Al}_{M} \mathrm{O}_{N}}-G_{\mathrm{TiC}} \\
& +M\left(3 \mu_{\mathrm{HCl}}-\frac{3}{2} \mu_{\mathrm{H}_{2}}-\mu_{\mathrm{AlCl}_{3}}\right) \\
& +N\left(\mu_{\mathrm{H}_{2}}-\mu_{\mathrm{H}_{2} \mathrm{O}}\right) .
\end{aligned}
$$

Here, $G_{\mathrm{TiC} / \mathrm{Al}_{M} \mathrm{O}_{N}}$ is the free energy of the film adsorbed on the TiC substrate, $G_{\mathrm{TiC}}$ is the free energy of the clean (relaxed) TiC substrate. The set of chemical potentials $\mu_{i}$ describe the Gibbs free energy of the various gases that contribute to the reaction.

The Gibbs free energy definition (8) is unambiguous and relevant for our nonequilibrium thermodynamic account of thin-film growth and interface formation. Of course, no direct reaction for the deposition described by (8) exists. However, stoichiometric combination of (7a) and $7 \mathrm{~b}$ effectively reduces to $4 \mathrm{~b}$. We treat $G_{\mathrm{r}}^{M, N}$ as a conservative function of the state variables $M$ and $N$ (that is independent of the details of the order of the deposition steps that result into an $\mathrm{Al}_{M} \mathrm{O}_{N}$ film). As a consequence, we can extract the values of $\Delta G_{\mathrm{r}}$ associated with the reaction that transforms a film characterised by $M^{\prime}$ and $N^{\prime}$ into a film that is characterised by $M$ and $N$ as difference between $G_{\mathrm{r}}^{M, N}$ and $G_{\mathrm{r}}^{M^{\prime}, N^{\prime}}$. These differences allow the direct calculation of the relative probabilities in the limit of dynamic equilibrium from our set of predictors (3).

We consider alumina films of the type $\mathrm{Al}_{4 t-4} \mathrm{O}_{6 t}$ (interface class A), $\mathrm{Al}_{4 t-2} \mathrm{O}_{6 t}$ (interface class $\mathrm{B}$ ) and $\mathrm{Al}_{4 t} \mathrm{O}_{6 t}$ (interface class $\mathrm{C}$ ), where $t$ is the thickness in terms of the number of $\mathrm{O}$ layers. Two times the reaction $7 \mathrm{a}$ thus corresponds to reaction $I$ and $I I$ in Figure 2 and six times the reaction (7b) corresponds to $I I I$. We note that, although $I$ and $I I$ are both formally described by the gas reaction (7a), they are different and possess different free energies of reaction since the substrates on which they occur and the final products are different.

We evaluate Gibbs free energies of reaction $G_{\mathrm{r}}^{M, N}$ as described in Refs. 33, 34, 39. The free energies of solid phases (substrate and potential thin films) are replaced by their DFT total energies, $G_{\text {solid }} \approx E_{\text {solid }} \cdot{ }^{4}$ For gases, we employ the ideal-gas approximation,

$$
\mu_{i}\left(T, p_{i}\right)=\epsilon_{i}+\Delta_{i}^{0}(T)+k_{B} T \ln \left(p_{i} / p^{0}\right) .
$$

4 In fact, we correct the total energies of the films by subtracting the strain energy of the stoichiometric part of the film, $E_{\text {film }} \rightarrow E_{\text {film }}-n_{\mathrm{Al}_{2} \mathrm{O}_{3}} \Delta_{\text {strain }}$, where $\Delta_{\text {strain }}$ is the is difference between the strained (due to the expansion to the TiC lattice in the interface plane) and the unstrained bulk alumina per stoichiometric unit.

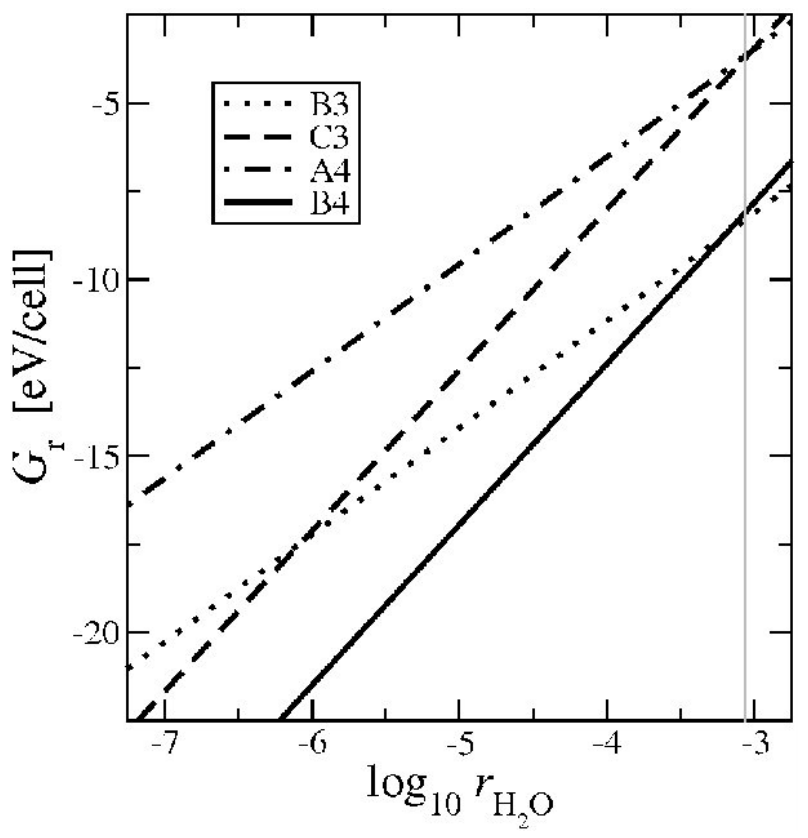

FIG. 4: Gibbs free energies of reaction $G_{\mathrm{r}}$ for formation of CVD thin-film alumina with different thickness and displaying different interfaces classes as functions of the scaled reaction rate $r_{\mathrm{H}_{2} \mathrm{O}}$. The vertical line corresponds to dynamic equilibrium in the water formation reaction. Parameters of Ref. 41 have been used for the composition of the environment. A deposition temperature of $T=1000{ }^{\circ} \mathrm{C}$, a pressure of $p=500$ mbar and $r_{\mathrm{Al}_{2} \mathrm{O}_{3}}=r_{\mathrm{H}_{2} \mathrm{O}} / 3.1$ were assumed. No qualitative changes arise when these parameters are varied in range typical for CVD of alumina [41]. The films are labeled according to their corresponding interface class (A, B or C) and their thickness in terms of $\mathrm{O}$ layers (here the cases for 3 and 4 layers are displayed).

Here $\epsilon_{i}$ is the DFT total energy of the gas (molecule) and $\Delta_{i}^{0}(T)$ is the temperature dependence of $\mu_{i}$ at a fixed pressure $p^{0}$. We use the values tabulated in Ref. 43 for $p^{0}=1 \mathrm{~atm}$. For the individual partial pressures $p_{i}$ we use steady-state pressures (6) specified by CVD process.

\section{RESULTS AND DISCUSSIONS}

\section{A. Gibbs free energies of reaction and growth}

In Figure 4 we plot the Gibbs free energies of reaction $G_{\mathrm{r}}$ [see (8)] for various thin-film alumina configurations on TiC. The films are labeled according to their corresponding interface class (A, B, C) and their thickness. We assume typical values for the the deposition temperature $T$, the total deposition pressure $p$ and for the concentrations $c_{i}$ of the different gases in the supply gas [41: $T=1000^{\circ} \mathrm{C}, p=500 \mathrm{mbar}, c_{\mathrm{AlCl}_{3}}=0.04, c_{\mathrm{CO}_{2}}=0.04$, and $\mathrm{H}_{2}$ constitutes the balance.

The vertical line corresponds to dynamic equilibrium in the water-producing step (4a). We emphasise that, in 
general, dynamic equilibrium in the water-producing step does not imply dynamic equilibrium in the alumina deposition. In the figure we have chosen $r_{\mathrm{Al}_{2} \mathrm{O}_{3}}=r_{\mathrm{H}_{2} \mathrm{O}} / 3.1$. For this value of $r_{\mathrm{Al}_{2} \mathrm{O}_{3}}$ the dynamic equilibria roughly coincide within our our approximation for the Gibbs free energy variation [36]. This follows from the observation that the values of $G_{\mathrm{r}}$ for the B3 and B4 films (which differ in thickness by one full layer or two stoichiometric units) are approximately equal on the vertical line. We note, however, that there is an uncertainty in this value of $r_{\mathrm{Al}_{2} \mathrm{O}_{3}}$ for coinciding dynamic equilibria. The reason is the uncertainty in the calculation of chemical potentials (in particular total energies of the molecules, see discussion in Ref. 39) and the uncertainty in the total energies of the films themselves. For the latter to be accurate we would have to make sure that their atomic structures are fully optimised. The geometries identified in Ref. 36 and used here are candidates for the optimised structures but not guaranteed to the optimised structures.

Figure 4 shows that, within the possible range of $r_{\mathrm{H}_{2} \mathrm{O}}$, all films have a negative value of $G_{\mathrm{r}}$. As a consequence all films are stable with respect to the substrate. This remains true also if we decrease the value of $r_{\mathrm{Al}_{2} \mathrm{O}_{3}}$.

The figure also demonstrates stoichiometric growth of alumina films. Stoichiometric growth corresponds to $\Delta G_{r}^{I}+\Delta G_{r}^{I I}+\Delta G_{r}^{I I I} \leq 0$. This condition is fulfilled as can be seen from the fact that the B4 film has lower free energy of reaction than the B3 film and that the former consists of two stoichiometric units of alumina more than the latter.

We emphasise that the Gibbs free energy variations shown in figure 4 directly reflect the closely related nature of process $I$ and $I I$. Process $I$ and $I I$ differ only in the solid reactants and solid products, the gaseous reactants and products are the same. In chemical reaction theory [40] we can express the Gibbs free energy of reaction as $\beta \Delta G_{\mathrm{r}}=\ln \Gamma_{f} / \Gamma_{b}=-\ln K_{\text {eq }}+\ln Q$. Here $K_{\mathrm{eq}}=k_{\mathrm{f}} / k_{\mathrm{b}}$ is the equilibrium constant of the reaction, $k_{\mathrm{f}}\left(k_{\mathrm{b}}\right)$ is the forward (backward) reaction rate constant and $Q$ is the reaction quotient (ratio of concentrations of gaseous products and of reactants). Since the gaseous reactants and products in process $I$ and $I I$ are identical, the reaction quotients for reaction $I$ and $I I$ are identical. Therefore, $\beta\left(\Delta G_{\mathrm{r}}^{I}-\Delta G_{\mathrm{r}}^{I I}\right)=\ln \left(K_{\text {eq }}^{I I} / K_{\text {eq }}^{I}\right)$ must be constant. In figure 4 we have

$$
\begin{aligned}
& \beta\left(\Delta G_{\mathrm{r}}^{I}-\Delta G_{\mathrm{r}}^{I I}\right) \\
& =\left[G_{r}(B 4)-G_{r}(A 4)\right]-\left[G_{r}(C 3)-G_{r}(B 3)\right] \\
& =\left[G_{r}(B 4)-G_{r}(C 3)\right]-\left[G_{r}(A 4)-G_{r}(B 3)\right] .
\end{aligned}
$$

We notice that the curves corresponding to B4 and C3 films are approximately parallel. The same applies for the curves corresponding to A4 and B3 films. Thus, both differences after the second equal sign in 10 are constant and the AIT-DG results can also be used to compute $\ln \left(K_{\mathrm{eq}}^{I I} / K_{\mathrm{eq}}^{I}\right)$.

\section{B. Thermodynamic analysis}

Figure 5 reports the calculated predictor (3) for the prevalence of interface class $\mathrm{A}$ and $\mathrm{C}$ relative to interface class $B$ as functions of the scaled reaction rate for water formation [see (4a)]. This predictor corresponds to the approximate relative steady-state probabilities (being exact in the limit of dynamic equilibrium in the alumina deposition). We have tested the quality of our predictor over a wide range of possible choices for unknown rate constants; we refer to the appendix for a more detailed presentation.

The vertical line corresponds to dynamic equilibrium in the water-producing step (4a). We assume the same values as before for the temperature and the concentrations of the different species in the supply gas [41. For the total deposition pressure and the reaction rate of alumina deposition we consider the following pairs of parameters, $p=500$ mbar and $r_{\mathrm{Al}_{2} \mathrm{O}_{3}}=r_{\mathrm{H}_{2} \mathrm{O}} / 3.1$, $p=10$ mbar and $r_{\mathrm{Al}_{2} \mathrm{O}_{3}}=r_{\mathrm{H}_{2} \mathrm{O}} / 3.1$ and $p=500 \mathrm{mbar}$ and $r_{\mathrm{Al}_{2} \mathrm{O}_{3}}=r_{\mathrm{H}_{2} \mathrm{O}} / 300$. The location of the vertical line is independent of these parameters (but depends on temperature).

We find that, in or close to dynamic equilibrium in the water producing step, the (approximate) probabilities for the prevalence of interface class $\mathrm{A}$ and $\mathrm{C}$ are much smaller than the probability for the prevalence of interface class $\mathrm{B}, P_{\mathrm{X}}<<P_{\mathrm{B}}(\mathrm{X}=\mathrm{A}$ or $\mathrm{C})$. As $r_{\mathrm{H}_{2} \mathrm{O}}$ decreases $P_{\mathrm{C}}$ increases and becomes larger than $P_{\mathrm{B}}$ at some point. The value of $P_{\mathrm{A}}$, on the other hand, decreases as $r_{\mathrm{H}_{2} \mathrm{O}}$ decreases. Thus, our results show that the nonbinding interface class $\mathrm{A}$ is not realised in CVD of alumina as described in Section IV. Instead, if reaction (4a) takes place sufficiently close to dynamic equilibrium, interface class B is predicted.

We note that it is by no means obvious that dynamic equilibrium is always maintained. If that was the case, $r_{\mathrm{Al}_{2} \mathrm{O}_{3}}$ would always assume its maximum value. Then, however, it would be possible to scale the absolute deposition rate $R_{\mathrm{Al}_{2} \mathrm{O}_{3}}=r_{\mathrm{Al}_{2} \mathrm{O}_{3}} \cdot R_{\mathrm{S}}$ to infinity simply by increasing the supply rate. It is against common sense to expect the deposition to remain in dynamic equilibrium as we increase the supply flux.

\section{Characterisation of interface adhesion}

Figure 6 details the electronic density at the interface class B. We have chosen the same isosurface level as in the top right panel of figure 1 . The Ti-O bonds show both ionic and covalent character. The density also reveals the partial covalent character of the otherwise highly ionic alumina itself. This covalent character can be seen by a small amount of electrons spilling over from neighbouring O layers.

A Bader analysis including core charges [44 46] shows that the ionicity of the Ti atoms is lower at the interface $\mathrm{B}\left(q_{\mathrm{Ti}}=1.71 e\right)$ than at the interface $\mathrm{A}\left(q_{\mathrm{Ti}}=1.88 \mathrm{e}\right.$, 


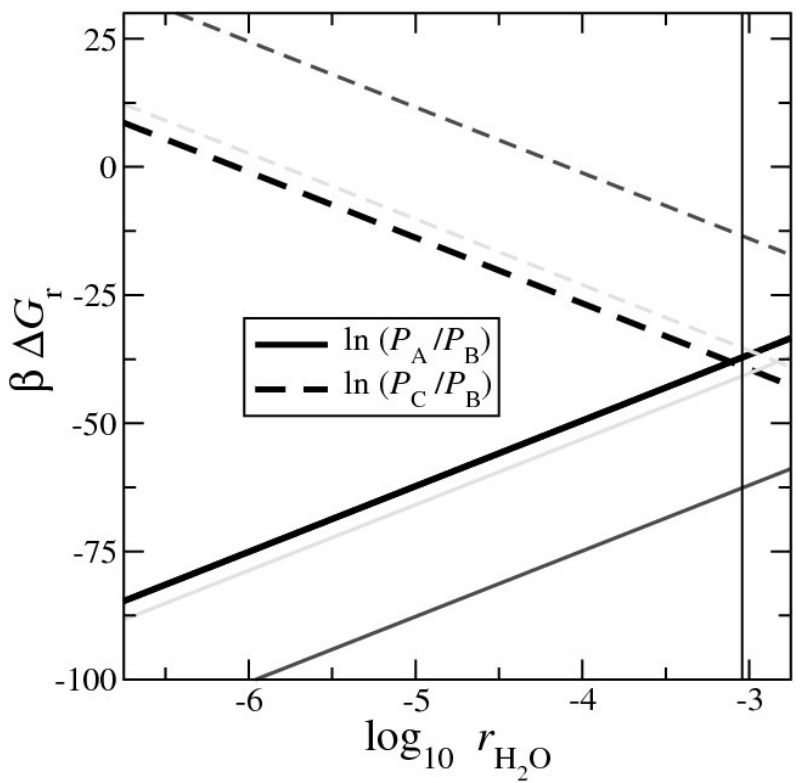

FIG. 5: Predictors for prevalence of interface class A (solid lines) and interface class $\mathrm{C}$ (dashed lines) as a functions of the scaled reaction rate $r_{\mathrm{H}_{2} \mathrm{O}}$ for water formation. The predictors are the approximate logarithm of the probability relative to the probability of prevalence of interface class $\mathrm{B}$. The vertical line limits $r_{\mathrm{H}_{2} \mathrm{O}}$ to the right and corresponds to dynamic equilibrium in the water formation, see 4a). Parameters of Ref. 41 have been used for the composition of the environment and a deposition temperature of $T=1000{ }^{\circ} \mathrm{C}$ was assumed. Black thick lines correspond to a deposition pressure of $p=500$ mbar and $r_{\mathrm{Al}_{2} \mathrm{O}_{3}}=r_{\mathrm{H}_{2} \mathrm{O}} / 3.1$, thin light lines to $p=10$ mbar and $r_{\mathrm{Al}_{2} \mathrm{O}_{3}}=r_{\mathrm{H}_{2} \mathrm{O}} / 3.1$, and thin dark lines to $p=500$ mbar and $r_{\mathrm{Al}_{2} \mathrm{O}_{3}}=r_{\mathrm{H}_{2} \mathrm{O}} / 300$. Close to dynamic equilibrium limit in reaction $4 \mathrm{a}$, we predict the highest prevalence for interface class B. As $r_{\mathrm{H}_{2} \mathrm{O}}$ decreases, the likelihood for the occurrence of interface class $\mathrm{C}$ increases. This likelihood strongly increases further with decreasing $r_{\mathrm{Al}_{2} \mathrm{O}_{3}}$ and also as the total deposition pressure increases. The nonbinding interface class A is highly unlikely to be created in the CVD process.

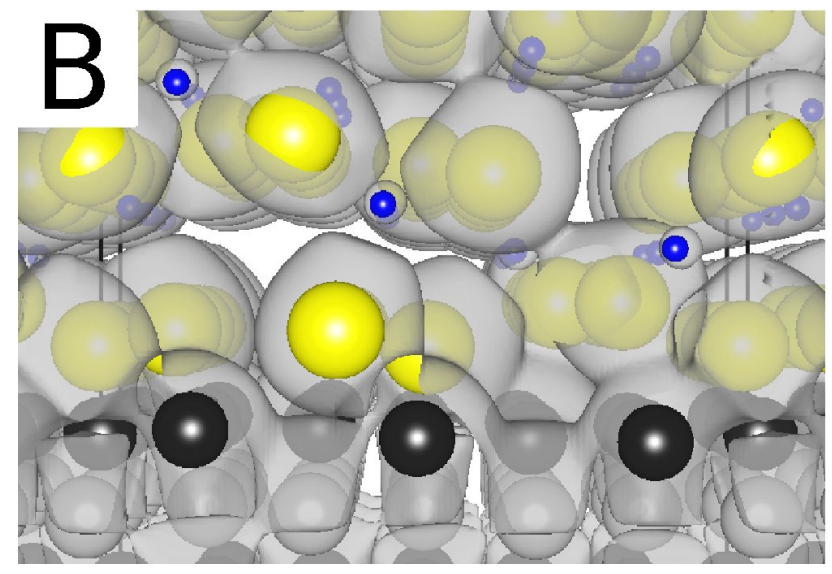

FIG. 6: (Colour online) Electron density at the interface B which shows the highest probability to be created in CVD of alumina on TiC. Same colour coding as in Fig. 1. top panels of figure 1). At the same time, the ionicity of the $\mathrm{O}$ atoms is higher $\left(q_{\mathrm{O}}=-1.46 e\right)$ at interface $\mathrm{B}$ than at interface $\mathrm{A}(q=-1.13 e)$. The Ti-O bond strength is lower at the interface $B$ than at the interface A. This is reflected by an increased Ti-O layer separation at interface $\mathrm{B}\left(l_{\mathrm{Ti}-\mathrm{O}}=1.48\right)$ with respect to that at interface $\mathrm{A}\left(l_{\mathrm{Ti}-\mathrm{O}}=0.88\right)$. However, the increased ionicity of the first $\mathrm{O}$ layer at interface $\mathrm{B}$ and the presence of $\mathrm{Al}$ ions between the first and the second $\mathrm{O}$ layers above the interface plane give rise to a strong overall interface adhesion. This adhesion is absent at interface A. Thus, the strong binding at the wear-resistant interface B that is most likely to be created in CVD of alumina arises from an interplay between a softening of the Ti-O bonding, an increased ionicity in the first $\mathrm{O}$ layer and a strong ionic binding in the alumina. The latter is supplemented by weaker covalent bonds between $\mathrm{O}$ layers in the alumina coating.

\section{SUMMARY AND CONCLUSION}

We have extended a recently proposed nonequilibrium method for ab initio thermodynamics of deposition growth from application to surfaces terminations [39] to prediction of thin-film and interface formation. Our results demonstrate that a careful treatment of deposition conditions in thin-film and interface modelling is crucial for understanding adhesion properties for CVD alumina on TiC. Assuming equilibrium between the oxide and $\mathrm{O}_{2}$ (as in 33 35]) results in prediction of a configuration that shows no binding across the interface (interface class A) 36, see top panel in Fig. 1] This is in conflict with the wear-resistant nature and industrial use of the material [37. In agreement with the wear-resistance of $\mathrm{TiC} /$ alumina coatings, we predict the deposition of strongly binding interface of type B (in or close dynamic equilibrium in the water forming step) or interface of type $\mathrm{C}$ (away from dynamic equilibrium).

We expect that a similar analysis will be necessary also for other buried interfaces that form during a deposition process in an environment that strongly differs from ambient conditions. The thermodynamic method used here only makes reference to the molecular species that are present (and directly relevant) during deposition. In contrast to equilibrium-thermodynamics approaches 33 35], this method is therefore not limited to oxides (although we here illustrate the method for a particular oxide) or materials that contain a constituent $\mathrm{X}$ for which a dimer $\mathrm{X}_{2}$ could serve as reference. Furthermore, the method allows for a search of conditions (supply gas compositions, deposition temperatures, deposition temperatures) that favour deposition of a pre-specified interface composition with desirable properties. Our results suggest that the nonequilibrium $a b$ initio thermodynamics method (Ref. 39] and present extension) can be useful in guiding experimental optimisation of present-day materials and design of novel such. 


\section{Acknowledgement}

We thank G. D. Mahan and C. Ruberto for valuable discussions. Support by the Swedish National Graduate School in Materials Science (NFSM), the Swedish Foundation for Strategic Research (SSF) through ATOMICS, the Swedish Research Council (VR) and the Swedish National Infrastructure for Computing (SNIC) is gratefully acknowledged.

\section{Appendix A: Determination of film prevalence}

The presentation of our results for the prevalence of the different alumina films rests on the analysis of the predictors $(3$. These predictors are strictly valid only under the assumption of dynamic equilibrium (in the alumina deposition). In this appendix we give a prescription of how the quality of these predictors can be tested with respect to the exact steady-state probability for the prevalence of different film classes.

We consider the exact steady-state ratio between the probability for $\mathrm{A}$ and $\mathrm{B}$, see equation (2). We introduce $x_{i}=\Gamma_{b}^{i} / \Gamma_{f}^{i}=\exp \left(\beta G_{r}^{i}\right)$ and $\alpha=x_{1}^{-1} x_{2}^{-1} x_{3}^{-1}$. The functions $\alpha$ and $x_{i}$ depend on $r_{\mathrm{H}_{2} \mathrm{O}}$ but are otherwise completely determined by our ab initio calculations (and the thermochemical data to determine the chemical potentials). In particular, the parameter $\alpha$ measures the departure from dynamic equilibrium; $\alpha=1$ corresponds to dynamic equilibrium, $\alpha>1$ corresponds to growth beyond dynamic equilibrium and $\alpha<1$ corresponds to evaporation. We furthermore introduce $F_{1}=\Gamma_{f}^{I} \Gamma_{f}^{I I}$ and $F_{2}=\Gamma_{f}^{I I I} \Gamma_{f}^{I}$. These functions also depend on $r_{\mathrm{H}_{2} \mathrm{O}}$ but require some additional parametrisation, see below.

Independent of the parametrisations, we can express the ratio of probabilities as

$$
\frac{P_{\mathrm{A}}}{P_{\mathrm{B}}}=\frac{x_{1} x_{2}+x_{1} F_{1} F_{2}+F_{2}}{\alpha^{-1} x_{1}^{-1} F_{2}+x_{2}+F_{1} F_{2}} .
$$

For the ratio between the probability for $\mathrm{C}$ and $\mathrm{B}$ we find

$$
\begin{aligned}
\frac{P_{C}}{P_{B}} & =\frac{\Gamma_{b}^{I} \Gamma_{b}^{I I I}+\Gamma_{f}^{I I} \Gamma_{b}^{I I I}+\Gamma_{f}^{I} \Gamma_{f}^{I I}}{\Gamma_{b}^{I I} \Gamma_{b}^{I I I}+\Gamma_{f}^{I} \Gamma_{b}^{I I}+\Gamma_{f}^{I} \Gamma_{f}^{I I I}} \\
& =\frac{F_{1} F_{2}+F_{2}+\alpha x_{1} x_{2}}{x_{2} F_{2}+\alpha x_{1} x_{2}^{-1}+\alpha x_{1} x_{2} F_{1} F_{2}}
\end{aligned}
$$

The quality of the approximate predictors 3 can be tested by evaluating (A1) and $\mathrm{A} 2$. The evaluation of A1 and A2 requires the specification of the functions $F_{1}$ and $F_{2}$. Here, we assume the following parametrisations,

$$
\begin{aligned}
& F_{1}=\frac{\Gamma_{f}^{I}}{\Gamma_{f}^{I I}}=\frac{k_{f}^{I}}{k_{f}^{I I}} \frac{\Pi_{i}\left[X_{i}^{I}\right]^{\nu_{i}^{I}}}{\Pi_{j}\left[X_{j}^{I I}\right]^{\nu_{j}^{I I}}} \\
& F_{2}=\frac{\Gamma_{f}^{I I I}}{\Gamma_{f}^{I}}=\frac{k_{f}^{I I I}}{\Pi_{f}^{I}} \frac{\Pi_{k}\left[X_{k}^{I I I}\right]^{\nu_{k}^{I I I}}}{\Pi_{i}\left[X_{i}^{I}\right]^{\nu_{i}^{I}}},
\end{aligned}
$$

where the $\nu_{i}$ can but do not need to be identical with the stoichiometric coefficients. We note that this parametrisation in only a crude approximation and that growth can, in general, be more complicated.

For alumina growth, the reactions $I$ and $I I$ are formally identical. The only difference is that they take place on different substrates and generate different solid products. Therefore, $F_{1}$ reduces to the ratio of the rate constants, which is constant. Reaction $I$ and $I I I$ are different and $F_{2}$ therefore depends on $r_{\mathrm{H}_{2} \mathrm{O}}$. Thus we have

$$
\begin{aligned}
& F_{1}=\frac{k_{f}^{I}}{k_{f}^{I I}} \\
& F_{2}=\frac{k_{f}^{I I I}}{k_{f}^{I}} \frac{\left[H_{2} O\right]^{m}\left[H_{2}\right]^{-n}}{\left[A l C l_{2}\right]^{r}\left[H_{2}\right]^{s}[H C l]^{-t}},
\end{aligned}
$$

where $m, n, r, s, t$ are all positive and of the order of 1 .

Since the rate constants $k_{f}^{I}, k_{f}^{I I}$, and $k_{f}^{I I I}$ are unknown, we have compared (3a) with A1 and (3c) with A2 for several choices of $m, n, r, s, t$ and a broad range of ratios of rate constants. We find that A2 is essentially described by (3c). For (A1) the approximation (3a) can deviate as $r_{\mathrm{H}_{2} \mathrm{O}}$ decreases but the qualitative result $\left(P_{\mathrm{A}}<<P_{\mathrm{B}}\right)$ is not affected. Moreover, the ratio $P_{\mathrm{A}} /\left(P_{\mathrm{B}}+P_{\mathrm{C}}\right)$ is always strongly suppressed.
[1] Wolf D and Yip S 1992 Materials interfaces: atomic-level structure and properties (Chapman \& Hall, London)

[2] Pacchioni G 2002 Surf. Sci. 5203

[3] Pukánszky B 2005 European Polymer Journal 41645

[4] Chalkera P R, Bulla S J and Rickerby D S 1991 Materials Science and Engineering: A 140583

[5] I. Chorkendorff and J. W. Niemantsverdriet, Concepts in
Modern Catalysis and Kinetics, 2nd ed. (WILEY-VCH, Weinheim, 2007).

[6] C. Stampfl, M. V. Ganduglia-Pirovano, K. Reuter, and M. Scheffler, Surf. Sci. 500, 368 (2002).

[7] Schönberger U, Andersen O K and Methfessel M 1992 Acta Metallurgica Et Materialia $40 \mathrm{~S} 1$

[8] Köstlmeier S, Elsässer C 2000 J. Phys.: Cond. Matter 
121209

[9] Schweinfest R, Köstlmeier S, Ernst F, Elsässer C, Wagner T and Finnis M W 2001 Philos. Mag. A 81927

[10] Hashibon A, Elsässer C and Rühle M 2005 Acta Mater. 535323

[11] S. V. Dudiy, J. Hartford, and B. I. Lundqvist 2000 Phys. Rev. Lett. 851898

[12] Wang X-G, Smith J R and Evans A 2002 Phys. Rev. Lett. 89286102

[13] Sinnott S B and Dickey E C 2003 Mater. Sci. Eng. R 43 1

[14] Beltrán J I and Muñoz M C 2008 Phys. Rev. B 78245417

[15] Noguera C, Finocchi F and Goniakowski J 2004 J. Phys.: Condens. Matter 16 S2509

[16] Christensen A and Carter E A 2001 J. Chem. Phys. 114 5816

[17] Jarvis E A and Carter E A 2002 Phys. Rev. B 66 100103(R)

[18] Johansson S A E and Wahnström G 2010 Phil. Mag. Lett. 90599

[19] Finnis M W 1996 J. Phys.: Condens. Matter 85811

[20] Lundqvist B I, Bogicevic A, Carling K, Dudiy S V, Gao S, Hartford J, Hyldgaard P, Jacobson N, Langreth D C, Lorente N, Ovesson S, Razaznejad B, Ruberto C, Rydberg H, Schröder E, Simak S I, Wahnström G and Yourdshahyan Y 2001 Surf. Sci. 493253

[21] Stampfl C, Ganduglia-Pirovano M V, Reuter K and Scheffler M 2002 Surf. Sci. 500368

[22] Nieminen R M 2001 J. Phys.: Condens. Matter 142859

[23] Fischer C, Tibbetts K, Morgan D and Ceder G $2006 \mathrm{Na}$ ture Materials 5641

[24] Nørskov J K, Bligaard T, Rossmeisl J and Christensen C H 2009 Nature Chemistry 137

[25] Kuehmann C J and Olson G B 2009 Materials Science and Technology 25472

[26] Chua A L-S, Benedek N A, Chen L, Finnis M W and Sutton A P 2010 Nature Materials 9418

[27] Mishin Y, Asta M and Li J 2010 Acta Materialia 581117

[28] Ziegler A, Idrobo J C, Cinibulk M K, Kisielowski C, Browning N D and Ritchie R O 2004 Science 3061768

[29] Holmström E, Olovsson W, Abrikosov I A, Niklasson A M N, Johansson B, Gorgoi M, Karis O, Svensson S, Schäfers F, Braun W, Öhrwall G, Andersson G, Mar- cellini M and Eberhardt W 2006 Phys. Rev. Lett. 97 266106

[30] Scheu C, Gao M, Oh S H, Dehm G, Klein S, Tomsia A $\mathrm{P}$ and Rühle M 2006 J. Mater. Sci. 415161

[31] Muller D A 2009 Nature Materials 8263

[32] see for example Rahman T S 2009 J. Phys.: Condens. Matter 21 080301, Borck Ø, Hyldgaard P and Schröder E 2007 Phys. Rev. B $\mathbf{7 5} 035403$

[33] Batyrev I G, Alavi A, and Finnis M W 2000 Phys. Rev. B 624698

[34] Reuter K and Scheffler M 2001 Phys. Rev. B 65035406

[35] Zhang W, Smith J R and Wang X-G 2004 Phys. Rev. B 70024103

[36] Rohrer J, Ruberto C and Hyldgaard P 2010 J. Phys.: Condens. Matter 22015004

[37] Halvarsson M, Norden H and Vuorinen S 1993 Surf. Coat. Technol. 61177

[38] Canovic S, Ruppi S, Rohrer J, Vojvodic A, Ruberto C, Hyldgaard P and Halvarsson M 2007 Surf. Coat. Technol. 202522

[39] Rohrer J and Hyldgaard P 2010 Phys. Rev. B 82045415

[40] Demirel Y Nonequilibrium Thermodynamics: Transport and Rate Processes in Physical, Chemical and Biological Systems, 2nd ed. (Elsevier, Amsterdam, 2007); Atkins $\mathrm{P}$ and de Paula J Atkins' Physical Chemistry, 7th ed. (Oxford University Press, 2002)

[41] Ruppi S and Larsson A 2001 Thin Solid Films 38850

[42] Hammer B, Nielsen O H, Mortensen J J, Bengtsson L, Hansen L B, Madsen A C E, Morikawa Y, Bligaard T, Christensen A, and Rossmeisl J available from https://wiki.fysik.dtu.dk/dacapo

[43] Chase M W Jr (Ed.) NIST-JANAF Thermochemical Tables (Washington, DC : American Chemical Society ; New York : American Institute of Physics for the National Institute of Standards and Technology, 1998).

[44] Henkelman G, Arnaldsson A and Jónsson A 2006 Comp. Matter Sci. 36354

[45] Berland K, Borck $\varnothing$ and Hyldgaard P 2010 submitted to Comp. Phys. Comm., see also arXiv:1007.3305v1 (condmat.mtrl-sci)

[46] Borck $\varnothing$ unpublished 